\title{
Dilated and contracted forms of primary endocardial fibroelastosis: a single fetal disease with two stages of development
}

\author{
A M Carceller, E Maroto, J-C Fouron
}

\begin{abstract}
Left ventricular endocardial fibroelastosis was diagnosed by echocardiographic scanning in a fetus at 20 weeks' gestation. Repeated prenatal examination over the next 20 weeks' gestation showed the development of the left ventricle from a chamber with a dilated cavity to a small cavity with a very thick wall. These findings were confirmed at necropsy and by the absence of other morphological anomalies.

The contracted form of primary fibroelastosis in the fetus must have followed an insult that prevented any further increase in the size of the left ventricular cavity.
\end{abstract}

The classification of primary endocardial fibroelastosis as either dilated or contracted depends on the size of the left ventricular cavity. ${ }^{1}$ So far only a few cases of the contracted form have been reported ${ }^{2}$ and these were exclusively in neonates. ${ }^{3}$ There are only a few reports of the detection of primary endocardial fibroelastosis in fetuses by echocardiography. ${ }^{45}$ In these cases, however, the dilated form of the disease only was reported in three infants at 28 , 35 , and 36 weeks' gestation respectively during a single evaluation. We used similar echocardiographic techniques to detect primary endocardial fibroelastosis in a fetus and follow the course of the disease over 20 weeks.

\section{Case report}

A 28 year old primigravida, without risk factors for congenital heart disease, was transferred to our fetal cardiology unit when a four chamber view of the fetal heart was found to be abnormal during routine obstetric ultrasonography at

Fetal Cardiology Unit, Section of Pediatric Cardiology, University of Montreal, SainteJustine Hospital, Montreal, Canada A M Carceller E Maroto J-C Fouron

Correspondence to Dr J-C Fouron, Service of Cardiology, Sainte-Justine ospital, 3175 Chemin Côte Ste-Catherine, Montreal, Quebec H3T 1C5, Canada. with poor mobility. Pulsed Doppler assessment of intracardiac flows showed a very low peak velocity and velocity time-integrals through the mitral and aortic valves (fig $1 B$ and $C$ ), whereas there was a considerable increase in pulmonary blood flow.

The mother was given digoxin immediately. After 24 hours, she was put on a maintenance dose of $0.5 \mathrm{mg}$ of digoxin. After a few days, the pericardial effusion disappeared but left ventricular contractility remained poor. Two months later, the fetal left atrium seemed to be slightly dilated and the interatrial septum bulged towards the right atrium (fig 2A). At 39 weeks' gestation, echocardiographic evaluations showed a decrease in the diameter of the left ventricular cavity which was associated with a thickening of the free wall with a very echogenic endocardium (fig 2B). At 40 weeks' gestation, a male infant weighing $3.25 \mathrm{~kg}$ was delivered vaginally. Apgar scores were 8 and 9 to 1 and 5 minutes respectively. Tests for viral infections were negative. Despite intensive cardiac support, the infant died seven days after birth.

Necropsy showed a thickened left ventricular wall with a small cavity lined by a white layer that was consistent with the diagnosis of endocardial fibroelastosis (fig 2C). The diagnosis was confirmed by microscopical examination (Weigert and trichrome stains). The mitral orifice was smaller than normal (diameter $0.6 \mathrm{~cm}$ ) but the mital valve leaflets were intact. The aortic valve was bicuspid with thin mobile cusps but the aortic root was only $0 \cdot 4$ $\mathrm{cm}$ in diameter. The ductus arteriosus and the foramen ovale were patent.

\section{Discussion}

Despite the obvious difference between the dilated and contracted forms of primary endocardial fibroelastosis we think that it is wrong to assume that there are two distinct diseases with different aetiologies. Our patient shows that in a fetus primary endocardial fibroelastosis can start as the dilated stage of the disease and progress to the contracted stage.

Though we did not seek histological evidence of fibroelastosis during gestation, the echocardiographic image of the endocardium was similar to the image at birth when there was histological confirmation of fibroelastosis. Because the fetal ventricles work in parallel, there are two possible courses after the onset of left ventricular failure early in gestation: either the right ventricle efficiently assumes almost 
Figure 1 (A)

Echocardiographic four chamber view of the heart at 20 weeks' gestation. The left ventricle $(L V)$ was dilated and a bright echogenic line was visible over the endocardium $(E)$. Pericardial fluid $(F)$ was also seen. $R V$, right ventricle. (B) Blood flow velocity profiles through the mitral and $(C)$ aortic valves. Left ventricular filling occurred chiefly during early diastole.
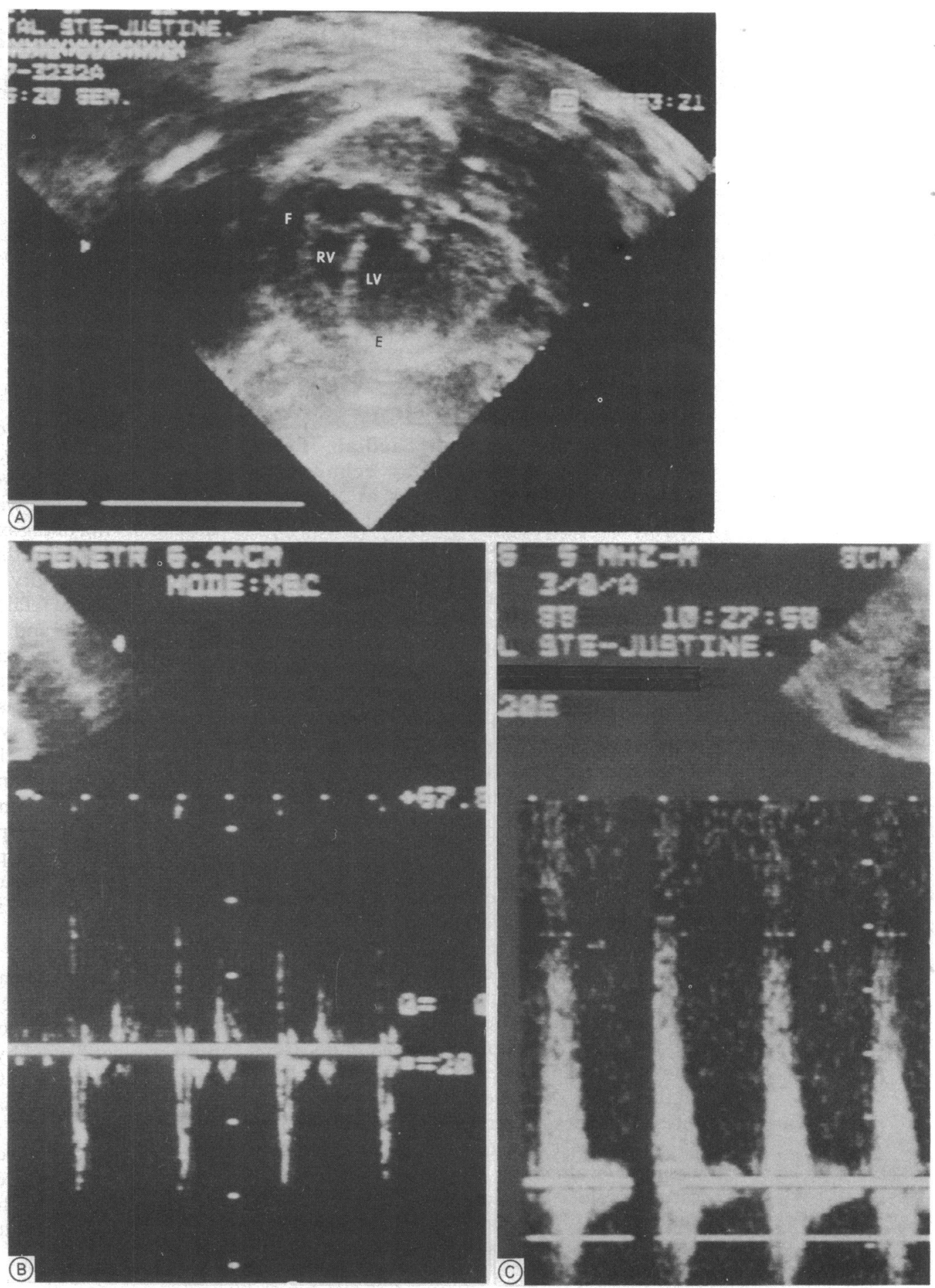

the entire circulatory load and fetal survival is possible or the right ventricle fails to cope with the new task and the fetus dies. The onset of endocardial fibroelastosis after birth also causes considerable left ventricular failure and because the ventricles now work in series survival depends upon the level of performance of both ventricles.

We first saw our patient at 20 weeks' gestation at an early stage in the development of fibroelastasis. The echocardiographic images were compatible with endocardial fibroelastosis, the left ventricle was hypotonic, dilated, and hydrops fetalis also indicated the failure of the right ventricle. Only rapid treatment of the mother with digoxin prevented the death of the fetus; and evidence of fluid retention disappeared. The ensuing events were predictableaccording to the dynamic concept of organic development ${ }^{6}$ - the very low blood flow into the left ventricle stopped the growth not only of the left ventricle but also the inflow and outflow valves and the ascending aorta. At birth, after 40 weeks' gestation the dimensions of the left-sided structures were those of a 28 week old fetus. These results support the speculation made by Neill and Ursell ${ }^{7}$ that just as the size of the foramen ovale can be used as a marker for intra-atrial flow in utero, so the size of the ascending aorta can be used as an indicator of intrauterine flow through the aortic valve. We believe that the contracted 

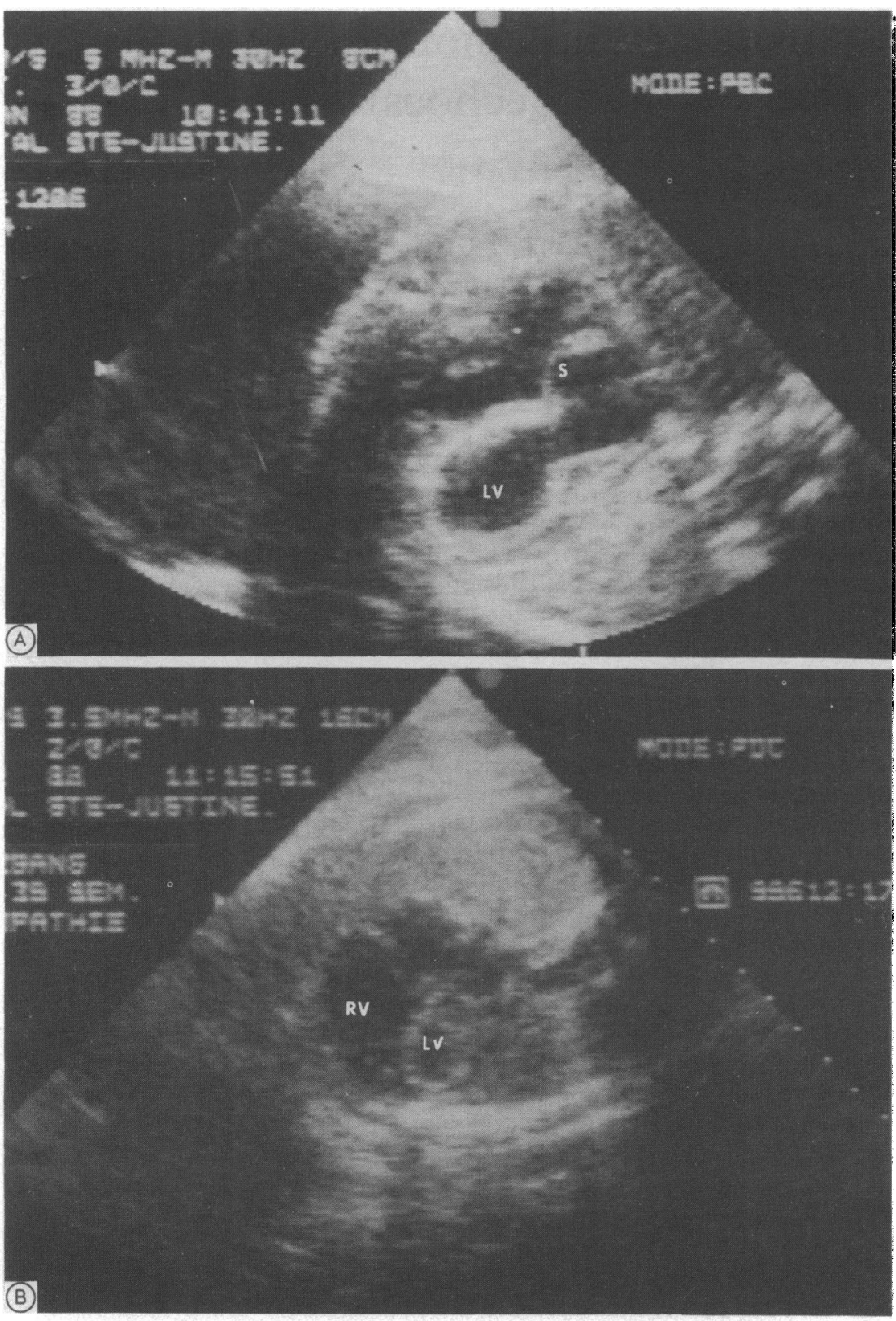

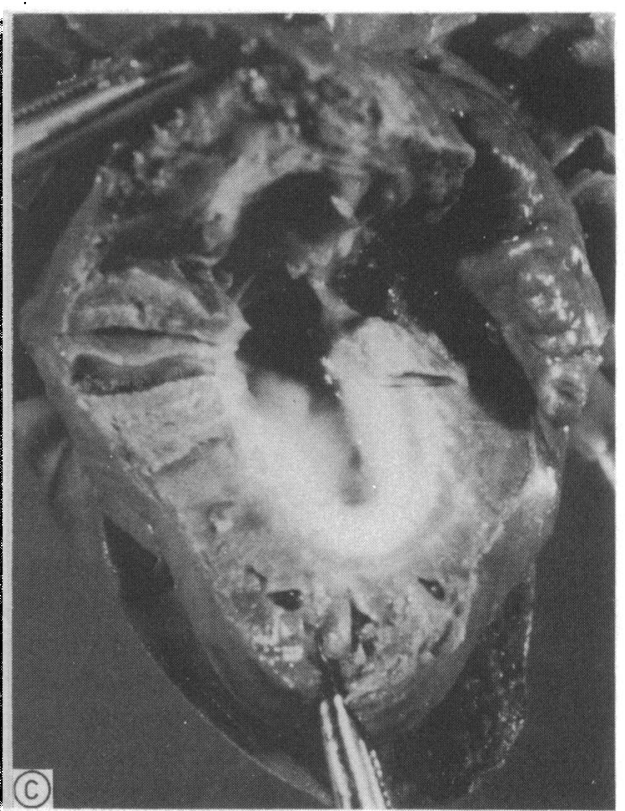

Figure 2 (A) An

echocardiographic view of

the interatrial septum $(S)$

bulging paradoxically

towards the right atrium

at 28 weeks' gestation. The

free wall of the left

ventricle $(L V)$ was thick

and the endocardium was

echogenic. (B) Four

chamber view at 39 weeks'

gestation showing a left

ventricular cavity ( $L V)$

with a smaller diameter.

$R V$, right ventricle. ( $C$ )

The heart showing a small

left ventricular cavity with

dense white endocardial

lining. The free wall was

very thick. form of primary fibroelastasis evolves from a dilated form of the disease in utero.

We thank Miss Susan P Drblik for her advice on this paper.

1 Edwards JE. Congenital malformations of the heart and great vessels. In: Gould SE, ed. Pathology of the heart. Springfield, Illinois: Charles C Thomas, 1953:420.

2 Ursell PC, Neill CA, Anderson RH, Siew YH, Becker AE Gerlis LM. Endocardial fibroelastosis and hypoplasia of the left ventricle in neonates without significant aortic stenosis. Br Heart $J$ 1984;51:492-7.

3 Blieden LC, Schneeweiss A, Deutsch V, Neufeld HN. Contracted form of endocardial fibroelastisis: angiographic diagnosis and necropsy confirmation. Pediatr Cardiol 1983;4:281-3.

4 Veille JC, Sivakoof M. Fetal echocardiographic signs of congenital endocardial fibroelastosis. Obstet Gynecol 1988; 72:219-22.

5 Bovicelli L, Picchio FM, Pilu G, et al. Prenatal diagnosis of endocardial fibroelastosis. Prenat Diagn 1984;4:67-72.

6 Rudolph AM, Heymann MA, Spitznas U. Hemodynamic considerations in the development of narrowing of the arta Am J Cardiol 1972:30:514-25.

7 Neill CA, Ursell P. Endocardial fibroelastosis and left heart hypoplasia revisited. Int J Cardiol 1984;5:547-50. 